\title{
Research Article \\ Ordinal Regression Based Subpixel Shift Estimation for Video Super-Resolution
}

\author{
Mithun Das Gupta, ${ }^{1}$ Shyamsundar Rajaram, ${ }^{1}$ Thomas S. Huang, ${ }^{1}$ and Nemanja Petrovic ${ }^{2}$ \\ ${ }^{1}$ Department of Electrical and Computer Engineering, University of Illinois, Urbana Champaign, IL 61801-2918, USA \\ ${ }^{2}$ Google Inc., 1440 Broadway, New York, NY 10018, USA
}

Received 2 October 2006; Accepted 3 May 2007

Recommended by Richard R. Schultz

We present a supervised learning-based approach for subpixel motion estimation which is then used to perform video superresolution. The novelty of this work is the formulation of the problem of subpixel motion estimation in a ranking framework. The ranking formulation is a variant of classification and regression formulation, in which the ordering present in class labels namely, the shift between patches is explicitly taken into account. Finally, we demonstrate the applicability of our approach on superresolving synthetically generated images with global subpixel shifts and enhancing real video frames by accounting for both local integer and subpixel shifts.

Copyright (C) 2007 Mithun Das Gupta et al. This is an open access article distributed under the Creative Commons Attribution License, which permits unrestricted use, distribution, and reproduction in any medium, provided the original work is properly cited.

\section{INTRODUCTION}

Shift estimation between two or more frames from a video has been of constant interest to researchers in computer vision. Need for accurate shift estimation arises from many practical situations. Applications, such as video frame registration, resolution enhancement, super-resolution, and optical-flow-based tracking, depend on reliable techniques for shift estimation for accuracy. Consequently, the accuracy of shift estimation methods is of utmost importance for these applications. Since the Lucas-Kanade [1] algorithm was proposed in 1981, image alignment has become one of the most significant contributions of computer vision. Applications of Lucas-Kanade image-alignment technique range from optical flow, tracking, layered motion to mosaic construction, medical image registration, and face coding. The principal idea of their technique was the introduction of imagegradients to infer the location of the target image patch in the subsequent frames which were best matches based on some arbitrary similarity metric. Many researchers have come up with refinements of their technique, to compute gradients in smarter ways, or to select the search region in smarter ways, but the principal idea has remained the same. Detailed reviews on motion estimation has been done by Aggarwal and Nandhakumar [2], Mitiche and Bouthemy [3], and Nagel [4]. Three main approaches to motion estimation can be identified as estimation based on spatial-gradient, image correlation, and regularization of spatiotemporal energy.

A closely related problem which has not yet received much focus in the literature is the problem of subpixel shift estimation which is harder than estimating shifts with pixel accuracy. The standard approach is to infer such shifts by interpolating to a higher resolution and then trying to estimate the shifts. These methods work relatively well when the subpixel shifts are global or are similar for large portions of the image. But if the shifts are varying drastically across small regions in the frames, then these techniques do not perform well. Patch-based techniques have an advantage, since the patch size can be adjusted based on variance of pixel intensities in a patch which can be a measure of the information in a patch. Most patch-based methods try to estimate the pixel shifts as well as subpixel shifts together, by using pyramid structures. One inherent drawback of such methods is that the neighborhood continuity constraints need to be satisfied at all levels of the pyramid. We try to answer a few of these issues in this work. We use Lucas-Kanade shift estimators [1] to estimate the pixel shifts and align the frames up to a pixel accuracy. We adopt a patch-based approach for subpixel shift estimation and estimate the subpixel shifts using a learning based framework upto a quarter pixel accuracy. Hence, each patch can be realigned up to a quarter pixel accuracy without affecting the continuity with its neighbors. 
The main contribution of this work is a learning-based method for subpixel estimation which falls under the category of supervised learning problems where the attributes are given by novel regression coefficient features representing two patches, and their corresponding label is the subpixel shift between them. Traditionally, the standard approach to solve such supervised learning problems which corresponds to learning the function mapping between the attributes and the label (subpixel shift) is to pose it as a multiclass classification problem or a regression problem. However, in our problem setting, there is a certain ordering present in the class labels, namely, the fractional shifts, which will not be captured by a classification or a regression approach. In this work, we exploit efficient learning algorithms that we proposed in our earlier work on ranking/ordinal regression [5] to perform subpixel shift estimation. The contribution of our earlier work was a set of efficient algorithms which learn ranking functions efficiently while explicitly capturing the inherent ordering present in the class labels. An elaborate description of the ranking algorithms is provided later.

The area of video super-resolution has gained steady interest from researchers over the past few years. The principal idea of super-resolving a video is to use information from temporal neighbors of a frame to help in generating the extra information needed for super-resolution. A certain number of neighbors from the past as well as the future are warped relative to the current frame so that they are aligned with the current frame. The warped images are now fused with the current frame to generate the super-resolved frame. This method is repeated for all the frames of the video, to get a super-resolved video. The warping of neighboring frames needs accurate shift estimation. Once the temporal neighbors are warped and aligned with the current frame, then the frames need to be combined for resolution enhancement. Most widely used combining methods are simple averaging and median operations, due to their simplicity and speed of implementation. Other sophisticated methods are mentioned in [6-10]. Accurate estimation of image motion has always been one of the most important bottlenecks of these techniques. In this work, we address the problem of accurate shift estimation by performing accurate estimation up to subpixel accuracy using the ranking/ordinal regression framework.

Our proposed approach is based on using learning-based methods for subpixel motion estimation. The basic idea behind our approach is that, once two image patches have been registered with respect to each other while accounting for integer shifts, the problem that remains to be solved is the estimation of subpixel shifts in the $x$ and $y$ directions. The subpixel shifts can then be used to align the patches in a higher resolution $[11,12]$. The subpixel estimation problem can be posed as a supervised learning problem in which the training data consists of pairs of image patches which are fractionally shifted with respect to each other by a known amount. The objective of the learning algorithm is to learn a function which learns the mapping from the features/attributes describing pairs of image patches to the corresponding subpixel shift between them while minimizing a certain loss function.
During the testing phase, given an unseen patch pair, the learned function is used to estimate the shift. The standard approach for solving the above supervised learning problem is to learn a multiclass classifier for learning the mapping from features to subpixel shifts. However, as shown in our earlier work [5], supervised learning problems in which the labels have an ordinal characteristic have to be treated differently by accounting for the ordering information present in the labels. Such an approach leads to an interesting ranking formulation which is termed ordinal regression in classical statistics literature. In the next few sections, we formalize the above ideas in a more general setting and develop algorithms for solving ranking problems.

The rest of the paper is organized as follows. Section 2 introduces notation and provides a formal description of the ranking model. In Section 3, we introduce the ranking model used in this work to pose the fractional shift estimation problem into an ordinal regression problem. We provide a detailed analysis of the complexity of the ranking model compared to the classification model. Next, we describe efficient schemes for performing ranking using standard classification algorithms. Section 4 reviews the use of motion estimation for performing super-resolution and Section 5 describes our super-resolution approach by accounting for subpixel shifts estimated using the ranking framework. In Section 6, we present experimental results of our subpixel shift estimation approach for performing super-resolution.

\section{NOTATIONS AND PROBLEM DEFINITION}

Consider a training sample of size $m$, say $S=\left\{\left(x_{1}, y_{1}\right)\right.$, $\left.\left(x_{2}, y_{2}\right), \ldots,\left(x_{m}, y_{m}\right)\right\}, x_{i} \in \mathbb{X}, y_{i} \in \mathbb{Y}$, where $\mathbb{X}$ is the domain representing the space of training examples and $\mathbb{Y}$ is the space from which labels are assigned to each example. We assume that $\mathbb{X}$ is the $n$-dimensional space of reals $\mathbb{R}^{n}$. Under this, for any $x_{i}, x_{j} \in \mathbb{X}$ we have $x_{i}-x_{j} \in \mathbb{X}$.

For the ranking problem, $\mathbb{Y}=\{1, \ldots, K\}$ where $K$ is the maximum rank that can be taken by any example. This is similar to the multiclass classification problem. However, the spirit of the ranking problem is very different. The ranks relate to the preference associated with an instance. Given an example with label $k$, all the examples with rank less than $k$ are ordered lesser and all the examples with rank more than $k$ are ordered higher. Such a relationship/viewpoint is not captured in the case of multiclass classification framework. In general, we will assume that $K$ (the maximum rank) is fixed for a given problem.

\section{THE RANKING MODEL}

In this work, we adopt a functional approach to solve the ranking problem. Given a set of data points $S$, we learn a ranker $f: \mathbb{X} \rightarrow \mathbb{Y}$. We assume that there exists an axis in some space, such that when data is projected onto this axis, the relative position of the data points captures the model of user preferences. In the ranking problem, we will treat $f$ to be a linear function $f\left(x_{i}\right)=h^{T} x_{i}$, whose value is the signed distance from some arbitrary hyperplane given by $h$. The information 
about the relative order of the data points will be captured by the distance from the hyperplane. In addition to learning $h$, we also learn $(K-1)$ thresholds corresponding to the different ranks that are assigned to the data. The learned classifier in this case is expressed as $\left(h ; \theta_{1}, \theta_{2}, \ldots, \theta_{K-1}\right)$ with the thresholds satisfying $\theta_{1}<\theta_{2}<\cdots<\theta_{K-1}$.

The ranking rule in this case is

$$
f\left(x_{i}\right)= \begin{cases}1 & \text { if } h^{T} x_{i}<\theta_{1} \\ \kappa & \text { if } \theta_{\kappa-1}<h^{T} x_{i}<\theta_{\kappa} \\ K & \text { if } \theta_{K-1}<h^{T} x_{i} .\end{cases}
$$

Although, this model of ranking may seem too simplistic, as we show in the next few sections, it is quiet powerful and we give an analysis relating Vapnik-Chervonenkis (VC) dimension of the learned classifier to what we call rankdimension of the data. We also show how one can extend the above framework to the case where learning needs to be done in a space different from the original space. In such a case, learning is done in some high-dimensional space by making use of kernels for the mapping.

\subsection{Complexity of ranking versus classification}

It has been argued [13] that the ranking problem is much harder than the classification problem. Although this is true in the particular view adopted by [13], in this paper we present an alternate viewpoint. We analyze the complexity of the ranking problem from the view of the $\mathrm{VC}$ dimension. We define the variant of the VC dimension, called rank dimension, for the ranking problem as follows: if the data points are ranked with respect to the value of the functional evaluated on a particular data point, then we say that the rank dimension of the functional is the maximum number of points that can be ranked in any arbitrary way using this functional.

Theorem 1. The rank dimension of a linear functional is same as its VC dimension. Following the notation given in Section 2, it holds for all $x_{i}, x_{j} \in X$, where $x_{i}-x_{j} \in \mathbb{X}$ and $x_{j}-x_{i} \in \mathbb{X}$.

Proof. Let us consider the case of linear classifier $h \in \mathbb{R}^{n}$. Say one observes a set of $m$ points $S=\left\{x_{1}, x_{2}, \ldots, x_{m}\right\}$, with the corresponding ranks $y_{1}, y_{2}, \ldots, y_{m}$.

Clearly if we can rank a set of $m$ points in any arbitrary way using a functional, then we can always shatter them (at the cost of one additional dimension corresponding to the threshold). Consider a subset $S_{0} \subset S$ such that we want to label all the points that belong to $S_{0}$ as negative and all the points that belong to $S$ but not to $S_{0}$ as positive (i.e., $S \backslash S_{0}$ ). Now if we rank all the points in such a way so that the rank of all the points in $S_{0}$ is less than the rank of all the points in $S \backslash S_{0}$, then we can do the classification by just thresholding based on the rank. This shows that the rank dimension of any functional cannot be more than the VC dimension of the same functional. We know that the $\mathrm{VC}$ dimension of a linear classifier in $n$-dimensional space is $n+1$. That is any set of $n+1$ points (assuming general positions) in $n$-dimensional space can be shattered by an $n$-dimensional linear classifier. Now we show that any set of $n+1$ points can be ranked in any arbitrary way using a linear classifier in $n$ dimensional space. Given any arbitrary ranking of the points, let's relabel the points such that $\operatorname{rank}\left(x_{1}\right)<\operatorname{rank}\left(x_{2}\right)<\cdots<\operatorname{rank}\left(x_{n+1}\right)$. Define a new set of points $S_{0}=\left\{0, x_{2}-x_{1}, x_{3}-x_{2}, \ldots, x_{n+1}-\right.$ $\left.x_{n}\right\}$. Now, if we label the points as $\{-1,1,1, \ldots, 1\}$, the cardinality of set $S_{0}$ is $n+1$ ( $n$-difference vectors and one 0 vector.) Also it is easy to see that all points in $S_{0}$ lie in $\mathbb{R}^{n}$. Now from the VC dimension theory, we know that there exists a linear classifier in $n$-dimensional space that can shatter $S_{0}$ according to the labeling given above. Let this linear classifier be $h$, with classification as $\operatorname{sign}\left(h^{T} x\right)$. Then for correct classification $h^{T}\left(x_{i}-x_{i-1}\right)>0 \Rightarrow h^{T} x_{i}>h^{T} x_{i-1}$. That indicates that the distance of the original points from the hyperplane does corresponds to the specified ranking. Hence, we have shown that any pair of $n+1$ points can be ranked in any arbitrary fashion by an $n$-dimensional classifier and at the same time we have also shown that the rank dimension cannot be more than the VC dimension. This shows that the rank dimension of any classifier is the same as its VC dimension.

This is a very interesting result as it shows that the complexity of the hypothesis space for the two problems is the same. However, as of now, we are not clear about the relation between the growth function for the two problems. Further, the relation between the computational complexity of the two problems has to be studied. We present two approaches to solve this problem. The first is referred to as the difference space approach while the second is referred to as the embedded space approach

\subsection{Difference space approach}

Given a training set $S$, define a new set $S_{d}$ of difference vectors $x_{i j}^{d}=x_{i}-x_{j}$; for all $i, j: y_{i} \neq y_{j}$ and their corresponding labels $y_{i j}^{d}=\operatorname{sign}\left(y_{i}-y_{j}\right)$. This leads to a dataset of size $O\left(m^{2}\right)$. Learning a linear classifier for this problem would be the same as learning a ranker $h$. Once such a ranker is learned, the thresholds for the ranking problem can easily be computed. This formulation is the same as the one proposed by [14]. Computational complexity of most learning algorithms, for example, naïve Bayes, depend linearly on the size of the training data and a quadratic increase in the size of the data will certainly make most of the existing algorithms impractical. Hence, we propose to generate difference vectors only among the adjacent rank classes. Formally, given a training set $S$, obtain a new set $S_{d}$ made up of difference vectors $x_{i j}^{d}=x_{i}-x_{j}$; for all $i, j: y_{i}=y_{j}+1$ and their corresponding labels $y_{i j}^{d}=+1$. This would result in a data-set with only positive examples. Again, most standard classification algorithms behave well if the number of positive examples is close to the number of negative examples. To get around this problem, once such a data set is obtained, multiply each example $x_{i j}^{d}$ and the corresponding label $y_{i j}^{d}$ by $q_{i j}$ where $q_{i j}$ is a random variable taking values $\{-1,1\}$ with equal probabilities. Clearly, learning a linear classifier over this data set will give a ranker $h$ which will be the same as the one obtained in the previous case. The size of the data set in this case is $O\left(\mathrm{~m}^{2} / K\right)$. For small $K$ (which is the case in most $K$-ranking problems) 


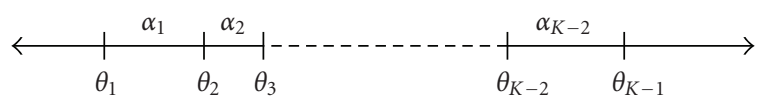

FIgURE 1: Ranking projection on the real line.

this is still too large to handle. Next, we present an efficient approach that specifically handles the ranking problem without exploding the size of the training data set.

\subsection{Embedded space approach}

In this section, we present a novel formulation that allows one to map a ranking problem to a standard classification problem without increasing the size of the data set. The embedded space approach presented in this section is similar in spirit to the model presented in [15], however as we will see shortly in our model, the dimension of the new space does not grow linearly as the one presented in their paper. Figure 1 graphically depicts the ranking framework. The distance from the hyperplane $h$ of a data point $x_{i}$ is mapped to a one-dimensional space. In this space, $\theta_{1}, \ldots, \theta_{K-1}$ are the different thresholds against which the distance is compared. Note that $h^{T} x_{i}$; for all $x_{i}$ having rank $\kappa$ results in a range represented by its left end point $\theta_{\kappa-1}$ and its right end point $\theta_{\kappa}$. Define $\alpha_{\kappa}=\theta_{\kappa+1}-\theta_{\kappa} ; 1 \leq \kappa \leq K-1$. For the data items belonging to rank 1 , there is no lower bound and for all the data items belonging to $\operatorname{rank} K$ there is no upper bound. By construction, it is easy to see that $\alpha_{\kappa}>0$; for all $\kappa$. Note that data point $x_{i}$ having rank $\kappa>1$ will satisfy (assuming $\alpha_{0}=0$ )

$$
\begin{gathered}
h^{T} x_{i}>\theta_{\kappa-1}, \\
h^{T} x_{i}+\alpha_{\kappa-1}>\theta_{\kappa}, \\
h^{T} x_{i}+\sum_{r=\kappa-1}^{K-2} \alpha_{r}>\theta_{K-1} .
\end{gathered}
$$

Similarly, for an example $x_{j}$ with $\operatorname{rank} \kappa<K$ (assuming $\theta_{K}=$ inf),

$$
\begin{gathered}
h^{T} x_{j}<\theta_{\kappa}, \\
h^{T} x_{j}+\alpha_{\kappa}<\theta_{\kappa+1}, \\
h^{T} x_{j}+\sum_{r=\kappa}^{K-2} \alpha_{r}<\theta_{K-1} .
\end{gathered}
$$

Based on this observation, define $\bar{h}=\left[h, \alpha_{1}, \alpha_{2}, \ldots, \alpha_{K-2}\right]$ and for an example $x_{j}$ with rank $1<\kappa<K$, define $\bar{x}_{j}^{+}, \bar{x}_{j}^{-}$ as $n+K-2$ dimensional vectors with

$$
\begin{aligned}
& \bar{x}_{j}^{+}[l]= \begin{cases}x_{j}[l], & 1 \leq l \leq n, \\
0, & n<l<n+\kappa-1, \\
1, & n+\kappa-1 \leq l \leq n+K-2,\end{cases} \\
& \bar{x}_{j}^{-}[l]= \begin{cases}x_{j}[l], & 1 \leq l \leq n, \\
0, & n<l<n+\kappa, \\
1, & n+\kappa \leq l \leq n+K-2 .\end{cases}
\end{aligned}
$$

For an example $x_{j}$ with rank $\kappa=1$, we define only $\bar{x}_{j}^{-}$as above and for an example with $\operatorname{rank} \kappa=K$, we define only $\bar{x}_{j}^{+}$again as above. This formulation assumes that $\theta_{K-1}=0$. It is easy to see that one can assume this without loss of generality (by increasing the dimension of $x$ by 1 one can get around this). Once we have defined $\bar{x}_{j}^{+}, \bar{x}_{j}^{-}$, the ranking problem simply reduces to learning a classifier $\bar{h}$ in $n+K-2$ dimensional space such that $\bar{h}^{T} \bar{x}_{j}^{+}>0$ and $\bar{h}^{T} \bar{x}_{j}^{-}<0$. This is a standard classification problem with at most $2 m$ training examples, half of which have label +1 (examples $\bar{x}_{j}^{+}$) and the rest have label -1 (examples $\bar{x}_{j}^{-}$). Even though, the overall dimension of the data points and the weight vector $h$ is increased by $K-2$, this representation limits the number of training data points to be $O(m)$. Note that although we have slightly increased the dimension by $K-2$, the number of parameters that need to be learned is still the same (the classifier and the thresholds). Interestingly, any linear classification method can now be used to solve this problem. It is easy to prove that if there exists a classifier that learns the above rule with no error on the training data, then all the $\alpha_{\kappa}$ s are always positive which is a requirement for the classification problem to be the same as the ranking problem. Next, we show how one can use kernel classifiers (SVM) to solve the ranking problem for data sets for which a linear ranker might not exist.

\subsection{Kernel classifiers: SVM}

In many real world problems, it may not be possible to come up with a linear function that would be powerful enough to learn the ranking of the data. In such a scenario, standard practice is to make use of kernels which allow nonlinear mapping of data. We will denote a kernel as $\mathcal{K}(\cdot, \cdot)=\phi^{T}(\cdot) \phi(\cdot)$ which corresponds to using the nonlinear mapping $\phi(\cdot)$ over the original feature vector.

Solving ranking problems. For solving the ranking problem, we have proposed the mapping given in Section 3.3, one has to be careful in using kernel classifiers with this mapping. To see this, note that if $x_{j}$ has rank $\kappa$, then $\bar{h}^{T} \bar{x}_{j}^{+}>0 \Rightarrow h^{T} x_{j}>$ $\theta_{\kappa-1} ; \bar{h}^{T} \bar{x}_{j}^{-}<0 \Rightarrow h^{T} x_{j}>\theta_{\kappa}$ but $\mathcal{K}\left(\bar{h}, \bar{x}_{j}^{+}\right)=\phi^{T}(\bar{h}) \phi\left(\bar{x}_{j}^{+}\right)>$ $0 \nRightarrow \phi^{T}(h) \phi\left(x_{j}\right)>\theta_{\kappa-1}$. This is again because of the nonlinearity of the mapping $\phi(\cdot)$. However, one can again get around this problem by defining a new kernel function. For a kernel function $\mathcal{K}$ and the corresponding mapping $\phi(\cdot)$, let us define a new kernel function $\overline{\mathcal{K}}$ and with the corresponding mapping $\bar{\phi}(\cdot)$ as

$$
\begin{aligned}
& \bar{\phi}(\bar{x})=[\phi(x), \bar{x}[n+1: n+K-2]], \\
& \bar{\phi}(\bar{h})=[\phi(h), \bar{h}[n+1: n+K-2]] .
\end{aligned}
$$

Note that, only the first $n$ dimensions of $\bar{x}$ corresponding to $x$ are projected to a higher dimensional space. The new kernel function can hence be decomposed into sum of two kernel functions where the first term is obtained by evaluation of kernel over the first $n$ dimensions of the vector and second 
term is obtained by evaluating a linear kernel over the remaining dimensions,

$$
\begin{aligned}
\mathcal{K}\left(\bar{x}_{i}, \bar{x}_{j}\right)= & \mathcal{K}\left(x_{i}, x_{j}\right) \\
& +\bar{x}_{i}[n+1: n+K-2]^{T} \bar{x}_{j}[n+1: n+K-2] .
\end{aligned}
$$

However, when using the SVM algorithm with kernels, one has to be careful while working in the embedded space. Learning algorithms typically minimize the norm $\|\bar{h}\|$ and not $\|h\|$ as should have been the case. In the next section, we introduce the problem of ordinal regression and show how one can get around this problem.

\subsection{Reduction to ordinal regression}

In this section, we show how one can actually get around the problem of minimizing $\|h\|$ as against minimizing $\|\bar{h}\|$. We want to solve the following problem

$$
\min \frac{1}{2}\|h\|^{2}, \quad \text { subject to } \bar{y}_{j}^{+/-}\left(\bar{h}^{T} \bar{x}_{j}^{+/-}+b\right)>0 .
$$

The inequality in the above formulation for $x_{j}$ with rank $y_{j}$ can be written as,

$$
\begin{aligned}
-b-\sum_{l=n+1}^{n+k-2} \bar{h}(l) \bar{x}_{j}^{-}(l) & =\theta_{y_{j}}>h^{T} x_{j} \\
& >-b-\sum_{l=n+1}^{n+k-2} \bar{h}(l) \bar{x}_{j}^{+}(l)=\theta_{y_{j}-1} .
\end{aligned}
$$

In this analysis, we will assume that with respect to threshold $\theta_{\kappa}$ 's, there is a margin of at least $\epsilon$ such that for any data point $x_{j}$ with corresponding rank $y_{j}$, we have

$$
\theta_{y_{j}-1}+\epsilon<h^{T} x_{j}, \quad 1<y_{j} \leq K .
$$

Now, the problem given in (7) can be reframed as

$$
\begin{gathered}
\min \frac{1}{2}\|h\|^{2}, \quad \text { subject to } h^{T} x_{j}<\theta_{y_{j}}, \forall 1 \leq y_{j}<K \\
h^{T} x_{j}>\theta_{y_{j}-1}+\epsilon ; \quad 1<y j \leq K .
\end{gathered}
$$

This leads to the following Lagrange formulation,

$$
\begin{aligned}
L_{P}= & \frac{1}{2}\|h\|^{2}+\sum_{j=1}^{m-m_{K}} \gamma_{j}^{+}\left(h^{T} x_{j}-\theta_{y_{j}}\right) \\
& +\sum_{j=m_{1}+1}^{m} \gamma_{j}^{-}\left(\theta_{y_{j}-1}+\epsilon-h^{T} x_{j}\right)-\sum_{j} \gamma_{j}^{+}-\sum_{j} \gamma_{j}^{-},
\end{aligned}
$$

where $m_{\kappa}$ refers to number of elements having rank $\kappa$. The ranker $h$ is obtained by minimizing the above cost function under the positivity constraints for $\gamma_{j}^{+}$and $\gamma_{j}^{-}$. Dual formulation $L_{d}$ of the above problem can be obtained by following the steps as in [16],

$$
L_{d}=-\frac{1}{2} \sum_{i=1}^{n} \sum_{j=1}^{n}\left(\gamma_{i}^{-}-\gamma_{i}^{+}\right)\left(\gamma_{j}^{-}-\gamma_{j}^{+}\right) \mathcal{K}\left(x_{i}, x_{j}\right)-\sum_{j} \gamma_{j}^{+}-\sum_{j} \gamma_{j}^{-}
$$

with constraints

$$
\sum_{p=m_{\kappa-1}+1}^{m_{\kappa}} \gamma_{p}^{+}=\sum_{p=m_{\kappa}+1}^{m_{\kappa+1}} \gamma_{p}^{-}, \quad \forall \kappa \in[2, K-1] .
$$

We have introduced $\gamma_{l}^{+}, \gamma_{m}^{-} \forall l \in\left[n_{K-1}+1, n_{K}\right]$ and $m \in$ $[1, n]$ for simplicity of notation. It is interesting to note that (12) has the same form as a regression problem. The value of $\theta_{\kappa}$ 's is obtained using Karush-Kuhn-Tucker (KKT) conditions.

\section{SUPER-RESOLUTION USING MOTION ESTIMATION}

In this section, we elaborate on the standard technique [17] that is used for video super-resolution by accounting for motion estimation. The outline of the super-resolution technique is as follows.

(1) Bilinearly interpolate the frames to double their original size.

(2) Warp $(t-n / 2)$ to $(t+n / 2)$ frames onto the reference frame $t$. This is one of the most important steps since it generates the extra information needed for superresolving the frames. The quality of the super-resolved frames depends on the accuracy of the image alignment techniques. A review of the different methods of performing this step is given in $[18,19]$.

(3) Obtain a robust estimate for the $t$ th frame. The way in which the extra information is combined to produce the frames plays an important role in determining their quality. Simple techniques like averaging or median operations to more complex techniques like covariance intersection can all be employed in this step. A few of the techniques for information fusion are [20-22].

(4) Iterate over all the frames (excluding the boundary frames).

(5) Repeat steps 2-4 until the estimates converge.

(6) Perform an optional deblurring operation on all the frames.

In this work, we follow the guidelines introducing our own algorithmic modules at appropriate places. For image registration and warping we use the hierarchical Lucas-Kanade method [1] as used in the original work by Baker and Kanade [17]. We use image patches of size $4 \times 4$ for all the experiments reported in this work. This particular size was found to be a good tradeoff between the gain attained by encoding information more than that contained by individual pixels and the smoothness introduced due to the patch size. As shown in some of our experiments, the subpixel shifts are still present after integer alignment and it is at this juncture that we perform our learning-based subpixel shift estimation algorithm. The details of our algorithm are provided in Section 5. For obtaining the robust estimate of the $t$ th frame, we note that there is a tradeoff between the simplicity of the technique and the time taken for estimation. We employ simple techniques like mean or median and find that the mean works quite well for the results reported in this work. As pointed in the original work by Baker and Kanade [17], simple mean works 
comparably against other complicated methods, and hence keeping the huge amount of video data in view, we adopt the simple mean as a method to combine the multiple frames. One point to note is that the 5th step in the algorithm which is essentially iterating over the whole algorithm is avoided for speed-up issues. All the results reported in this work are run just for one iteration. Also the last step (6) has been avoided in all our experiments. We take the liberty of omitting the last step from all our results since this step is independent of the algorithm used for the previous steps, and this work is principally focussed on performing the shift estimation and not on spatial domain deblurring techniques.

\section{SUBPIXEL SHIFT ESTIMATION}

Subpixel shift estimation involves identifying the shift in the $x$ and $y$ directions between two patches wherein, we assume that they have already been aligned in such a way that there are no integer shifts between them. Traditionally, this problem has been solved using resolution pyramids in which the subpixel shift problem is posed as an integer shift problem in higher resolution. However, such a technique is limited by the interpolation algorithm used for increasing the resolution. In this work, we adopt a learning strategy, namely, the ranking framework discussed earlier for estimating the subpixel shifts without increasing resolution. We note that the notion of preference modeled by the ranking framework, corresponds to the subpixel shift between two patches, say in the $x$ direction. Consider three patches denoted by $p_{1}, p_{2}$, and $p_{3}$ and let the shift between $p_{2}$ and $p_{1}$ be a quarter pixel shift and the shift between $p_{3}$ and $p_{1}$ be a half a pixel shift in the $x$ direction. The ranking framework accounts for the ordering information, that is, $p_{1}$ is closer to $p_{2}$ than $p_{3}$. Such ordering information is not captured if we use a multiclass classifier. The estimation problem becomes unrealistic when posed as a regression problem because it imposes a metric on the ranker output.

\subsection{Polar coordinates}

The subpixel shift estimation problem involves estimating two different rankers which capture the shifts in the $x$ and $y$ directions. However, we note that the two ranking problems are interrelated and treating them independently results in bad empirical performance. Hence, we decouple the relation present in the Euclidean setting to an extent by posing the shift estimation problem in the polar domain which corresponds to estimating the shift in the radial direction and angular direction. In the radial direction, the learning problem falls under the category of the ranking formulation elaborated in Section 1. However, estimating shifts in the angular direction leads to a different formulation that we term circular ordinality. Such a behavior arises naturally because of the equivalence of an angular shift of 0 and $2 \pi$. Modeling such behavior explicitly needs defining the notion of an angular margin introducing nonlinearities in the cost function which is hard to optimize. We overcome the above problem, by a two step algorithm. The first step involves a classifier that

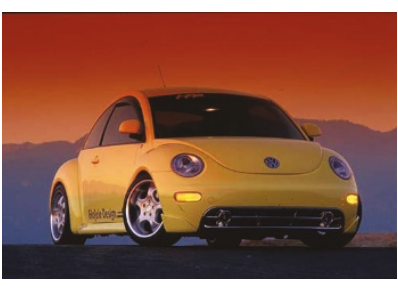

(a)

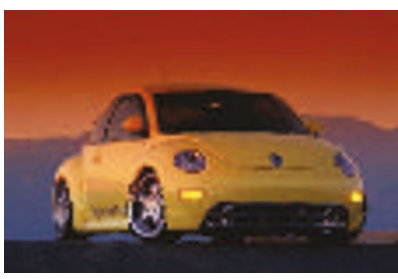

(c)

(e)

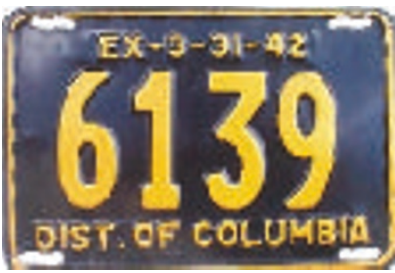

(g)

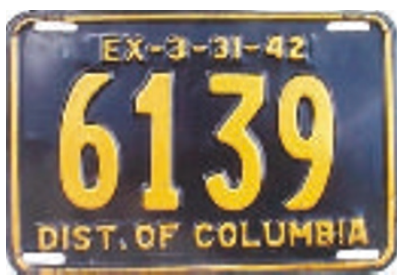

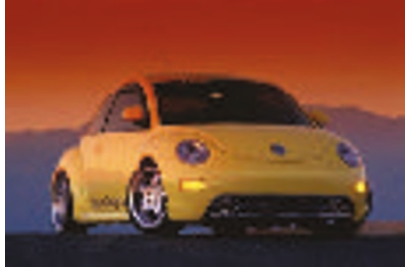

(b)

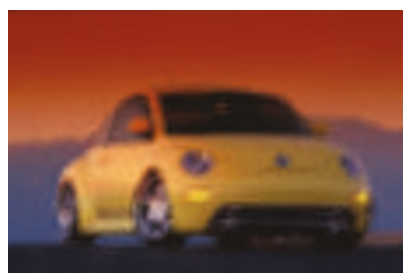

(d)

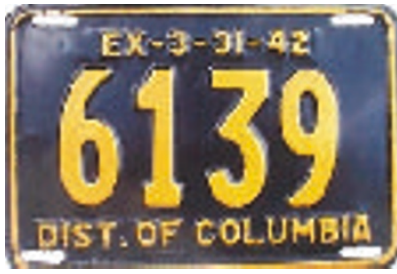

(f)

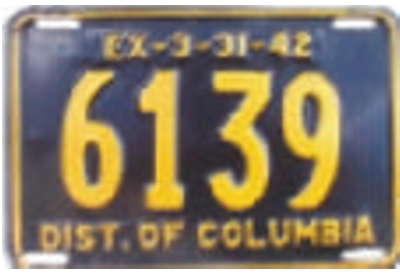

(h)
FIGURE 2: Image super-resolution. For each class, clockwise from top left: (a), (e) original image, (b), (f) quarter pixel accuracy, (d), (h) integer-pixel accuracy, (c), (g) half-pixel accuracy.

identifies the angular shift between two patches as either being in the upper half space (which corresponds to an angular shift of $0-\pi$ ) or in the lower half space (which corresponds to an angular shift of $\pi-2 \pi$ ). The second step involves identifying the angular shift in the relevant half space which can be solved using the ranking framework. In this work, we discretize the angular space uniformly into eight segments.

\subsection{Regression coefficients as features}

An important component of modeling is to identify/ construct features/attributes that efficiently represent the input space in an informative way such that they aid in solving the learning problem well. Another novelty of this work is a set of novel features for representing patch pairs. Consider two patches $\mathfrak{p}_{i}$ and $\mathfrak{p}_{j}$, where we denote the pixels in the patches as $\mathfrak{p}_{i k}$, and let there be $\mathcal{P}$ pixels in each patch. 


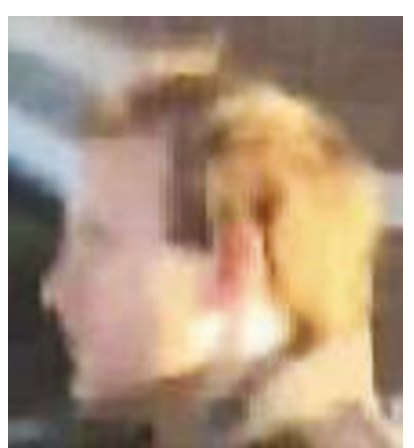

(a)

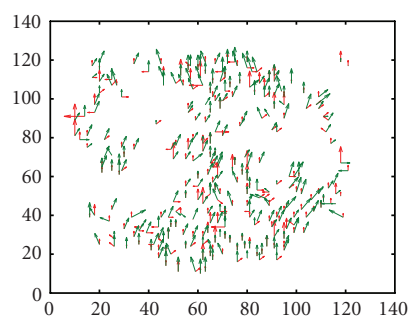

(e)

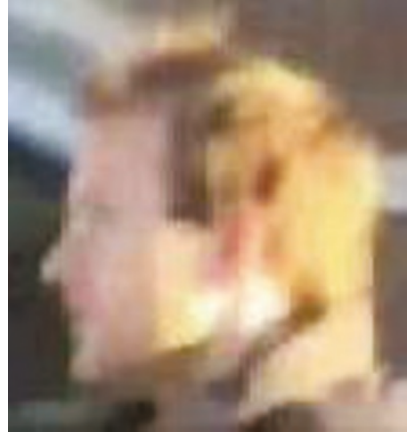

(b)

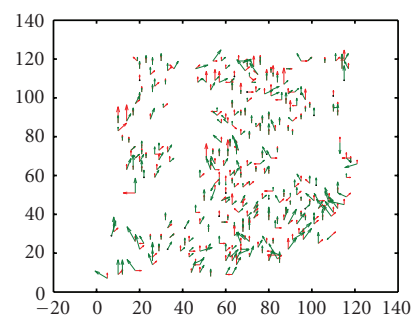

(f)

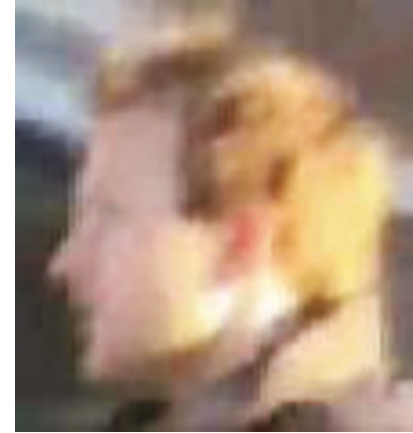

(c)

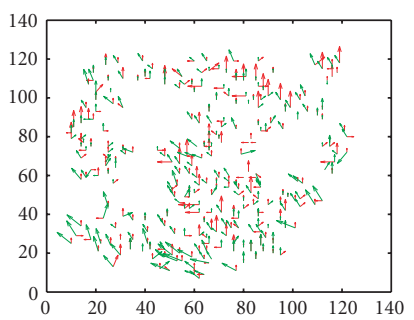

(g)

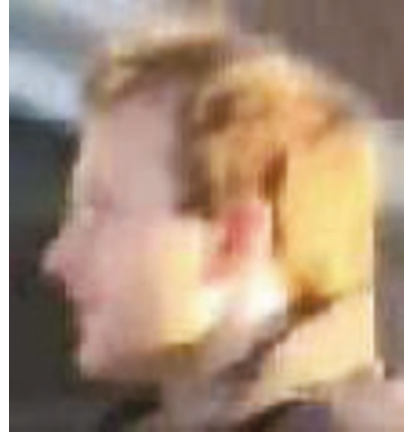

(d)

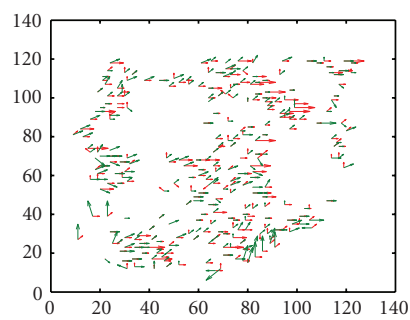

(h)

Figure 3: Top row: frames from the video. Bottom row: scaled flow vectors. Green: Lucas-Kanade. Red: our method.

We denote the set of adjacent neighbors of pixel location $k$ as $\mathcal{N}(k)$. We exploit the nature of subpixel shifts to model every nonboundary pixel in the patch $\mathfrak{p}_{j k}$, as a linear combination of the pixels $\mathfrak{p}_{i l}$ in the patch $\mathfrak{p}_{i}$ where $l \in\{\mathcal{N}(k) \cup k\}$. The above step results in a set of linear equations given by

$$
\mathfrak{p}_{j k}=w_{i j}^{T} \mathfrak{p}_{i l}, \quad l \in\{\mathcal{N}(k) \cup k\},
$$

where $w_{i j}$ represents the weight vector and $k$ indicates one of the nonboundary pixels in the patch $\mathfrak{p}_{j}$. Further, we note that the weight vector is invariant of $k$. We solve the above linear regression problem in a least mean square error sense to obtain the regression coefficients vector $w_{i j}$. These regression coefficients are used to represent the patch pair $\mathfrak{p}_{i}$ and $\mathfrak{p}_{j}$. Higher order models can be used to model the patch dependencies. Potential candidates to replace the linear predictors can be median filter-based predictors [23] or hybrid filters [24].

\section{EXPERIMENTAL RESULTS}

The experiments that we performed to demonstrate the applicability of our approach can be broadly classified into two categories. The first experiment involves estimating global motion for static images where the motion is generated exclusively by camera motion and the scene is assumed to be fixed. We estimate the amount of subpixel shift within consecutive frames and project the frames onto a higher resolution grid by accounting for the subpixel shifts. The unknown pixels on the grid are then interpolated to generate highresolution images. In the second experiment, we demonstrate the applicability of our approach on video frames which have a moving foreground object and varying local motion across different parts of the frame. We use subpixel alignment techniques to generate the super-resolved video as elaborated in Section 4.

\subsection{Global subpixel motion}

In this subsection, we investigate image super-resolution with global subpixel shift estimation in which we synthetically generate the images by simulating camera shift against a static background. We test our method for 3 different categories of images, namely, cars, license plates, and human subjects. The training data used to learn the radial direction ranker and the classifier and ranker for angular direction estimation includes a mix of images belonging to different categories from the Corel data set. The technique of estimating subpixel shifts is then used to perform static image super-resolution. We used the traditional spatial alignment method, wherein multiple low-resolution images are aligned on a higher resolution grid. The unknown pixels remaining on the grid are generated by bicubic interpolation. Since the resolution improvement quality depends on the number of grid locations that can be filled accurately, without interpolation, subpixel accuracies perform significantly better than accounting for pure integer shifts. Also, the higher the resolution of the subpixel estimation is the better the results would be. We perform these experiments for the 3 classes of images mentioned above. The results are shown in (Figure 2). Clearly, our method (quarter pixel accuracy) and half-pixel accuracy are better than integer pixel accuracy. Note the edges of the car bonnet or the edges of the digits in the plate. 


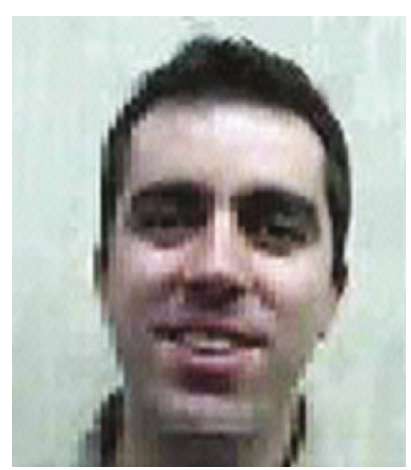

(a)

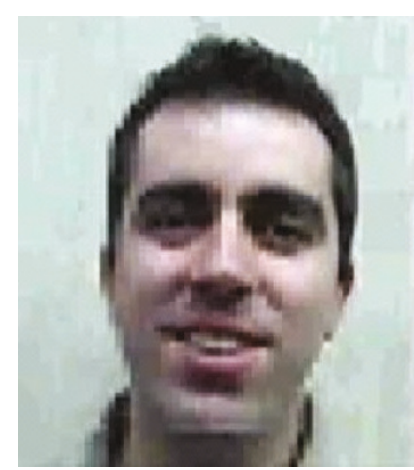

(b)

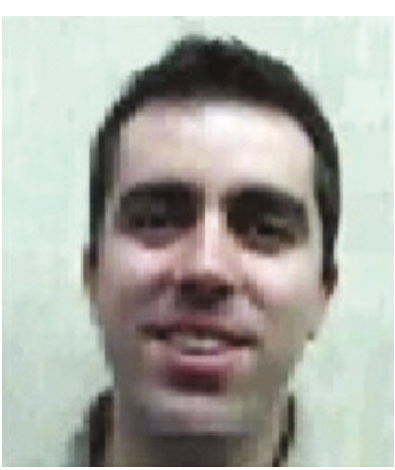

(c)

FIGURE 4: Left to right: (a) original scaled frame, (b) bicubic interpolation, (c) our method.

\subsection{Local subpixel motion}

In this subsection we report video super-resolution results by estimating subpixel shifts of face videos. All the video are shot with a Canon SD 450 camera. The frames are downsampled to create the low-resolution inputs to our system.

In the first set of experiments, we verify the claim that subpixel shifts still remain intact even after the frames have been stabilized with respect to their temporal neighbors. For this experiment, we took a video of a walking person and stabilized the video using the algorithm proposed in $[25,26]$. We use feature tracking to obtain the flow vectors and then use our method to obtain the subpixel shifts in addition to the flow vectors. We use Lucas-Kanade [1] to obtain the optical flow vectors. The results are shown in Figure 3.

The final set of results show sequence of frames from a face video. Each frame has been super-resolved using the approach elaborated in Section 4. We compare our results against bicubic interpolation of the frames as shown in Figure 4. Note that we do not perform the addition deblurring step which is commonly performed in other video super-resolution algorithms. The results clearly indicate the enhancement gain while performing motion and subpixel shift estimation.

\section{CONCLUSION AND FUTURE WORK}

We have presented a learning-based algorithm for estimating subpixel shifts in a patch based setting. The learning based algorithm falls under the class of ranking problems in which the ordering of the class labels is explicitly accounted for which results in better performance over standard classification and regression approaches. The ranking approach for subpixel shift estimation is used to perform super-resolution of images which have undergone a global subpixel shift and enhancement of video frames which have undergone both integer and subpixel shifts.

As mentioned earlier, higher order nonlinear models for patch dependencies can be used to generate the features for the determination of the azimuth angle. In the future, we plan to use the subpixel shift estimation approach for other applications, namely, motion tracking, layered motion, mo- saic construction, medical image registration, and face coding. In the local subpixel shift estimation scenario, further super-resolution can be performed by aligning pixels in a higher-dimensional grid.

\section{ACKNOWLEDGMENT}

This work was supported in part by Disruptive Technology Office (DTO) under Contract NBCHC060160.

\section{REFERENCES}

[1] B. Lucas and T. Kanade, "An iterative image registration technique with an application to stereo vision," in Proceedings of the 7th International Joint Conference on Artificial Intelligence (IJCAI '81), pp. 674-679, Vancouver, BC, Canada, August 1981.

[2] J. K. Aggarwal and N. Nandhakumar, "On the computation of motion from sequences of images-a review," Proceedings of the IEEE, vol. 76, no. 8, pp. 917-935, 1988.

[3] A. Mitiche and P. Bouthemy, "Computation and analysis of image motion: a synopsis of current problems and methods," International Journal of Computer Vision, vol. 19, no. 1, pp. 2955, 1996.

[4] H.-H. Nagel, "Image sequence evaluation: 30 years and still going strong," in Proceedings of the 15th International Conference on Pattern Recognition (ICPR '00), vol. 1, pp. 149-158, Barcelona, Spain, September 2000.

[5] S. Rajaram, A. Garg, X. S. Zhou, and T. S. Huang, "Classification approach towards ranking and sorting problems," in Proceedings of the 14th European Conference on Machine Learning (ECML '03), pp. 301-312, Cavtat-Dubrovnik, Croatia, September 2003.

[6] M.-C. Chiang and T. Boult, "Efficient image warping and super-resolution," in Proceedings of the 3rd Workshop on Applications of Computer Vision (WACV'96), pp. 56-61, Sarasota, Fla, USA, December 1996.

[7] F. Dellaert, S. Thrun, and C. Thorpe, "Jacobian images of super-resolved texture maps for model-based motion estimation and tracking," in Proceedings of the 4th IEEE Workshop on Applications of Computer Vision (WACV'98), pp. 2-7, Princeton, NJ, USA, October 1998. 
[8] M. Elad and A. Feuer, "Super-resolution restoration of an image sequence: adaptive filtering approach," IEEE Transactions on Image Processing, vol. 8, no. 3, pp. 387-395, 1999.

[9] R. C. Hardie, K. J. Barnard, and E. E. Armstrong, "Joint MAP registration and high-resolution image estimation using a sequence of undersampled images," IEEE Transactions on Image Processing, vol. 6, no. 12, pp. 1621-1633, 1997.

[10] A. J. Patti, M. I. Sezan, and A. M. Tekalp, "Super-resolution video reconstruction with arbitrary sampling lattices and nonzero aperture time," IEEE Transactions on Image Processing, vol. 6, no. 8, pp. 1064-1076, 1997.

[11] T. S. Huang and R. Tsai, "Multi-frame image restoration and registration," in Advances in Computer Vision and Image Processing, T. S. Huang, Ed., vol. 1, pp. 317-339, JAI Press, Greenwich, Conn, USA, 1984.

[12] S. P. Kim, N. K. Bose, and H. M. Valenzuela, "Recursive reconstruction of high resolution image from noisy undersampled multiframes," IEEE Transactions on Acoustics, Speech, and Signal Processing, vol. 38, no. 6, pp. 1013-1027, 1990.

[13] W. W. Cohen, R. E. Schapire, and Y. Singer, "Learning to order things," Journal of Artificial Intelligence Research, vol. 10, pp. 243-270, 1999.

[14] R. Herbrich, T. Graepel, and K. Obermayer, "Large margin rank boundaries for ordinal regression," in Advances in Large Margin Classifiers, pp. 115-132, MIT Press, Cambridge, Mass, USA, 2000.

[15] D. Roth, S. Har-Paled, and D. Zimak, "Constraint classification: a new approach to multiclass classification," in Proceedings of the 13th Interntional Conference on Algorithmic Learning Theory (ALT '02), pp. 365-379, Lübeck, Germany, November 2002.

[16] A. Smola and B. Schlkopf, "A tutorial on support vector regression," Tech. Rep. NC2-TR-1998-030, Neural and Computational Learning 2 (NeuroCOLT2), London, UK, 1998.

[17] S. Baker and T. Kanade, "Super-resolution optical flow," Tech. Rep. CMU-RI-TR-99-36, Carnegie Mellon University, Pittsburgh, Pa, USA, 1999.

[18] S. Baker and I. Matthews, "Lucas-kanade 20 years on: a unifying framework," International Journal of Computer Vision, vol. 56, no. 3, pp. 221-255, 2004.

[19] G. Wolberg, Digital Image Warping, IEEE Computer Society Press, Los Alamitos, Calif, USA, 1992.

[20] X. Li, Y. Zhu, and C. Han, "Unified optimal linear estimation fusion-I: unified models and fusion rules," in Proceedings of the 3rd International Conference on Information Fusion (FUSION '00), vol. 1, pp. 10-17, Paris, France, July 2000.

[21] S. Julier and J. Uhlmann, "A non-divergent estimation algorithm in the presence of unknown correlations," in Proceedings of the IEEE American Control Conference (ACC'97), vol. 4, pp. 2369-2373, Alberquerque, NM, USA, June 1997.

[22] D. Comaniciu, "Nonparametric information fusion for motion estimation," in Proceedings of the IEEE Computer Society Conference on Computer Vision and Pattern Recognition (CVPR '03), vol. 1, pp. 59-66, Madison, Wis, USA, June 2003.

[23] T. C. Aysal and K. E. Barner, "Quadratic weighted median filters for edge enhancement of noisy images," IEEE Transactions on Image Processing, vol. 15, no. 11, pp. 3294-3310, 2006.

[24] T. C. Aysal and K. E. Barner, "Hybrid polynomial filters for Gaussian and non-Gaussian noise environments," IEEE Transactions on Signal Processing, vol. 54, no. 12, pp. 4644-4661, 2006.

[25] N. Petrovic, N. Jojic, and T. S. Huang, "Hierarchical video clustering," in Proceedings of the 6th IEEE Workshop on Multi- media Signal Processing (MMSP '04), pp. 423-426, Siena, Italy, September 2004.

[26] N. Jojic, N. Petrovic, B. J. Frey, and T. S. Huang, “Transformed hidden Markov models: estimating mixture models of images and inferring spatial transformations in video sequences," in Proceedings of the IEEE Computer Society Conference on Computer Vision and Pattern Recognition (CVPR '00), vol. 2, pp. 26-33, Hilton Head, SC, USA, June 2000.

Mithun Das Gupta received his B.S. degree in instrumentation engineering from Indian Institute of Technology, Kharagpur in 2001, and his M.S. degree in electrical engineering from University of Illinois at Urbana Champaign in 2003. He is currently pursuing his Ph.D. degree under the guidance of Professor Thomas S. Huang at the University of Illinois at Urbana Champaign. His research interests include learning-based methods for image and video understanding and enhancement.

Shyamsundar Rajaram received the B.S. degree in electrical engineering from the University of Madras, India, in 2000, and the M.S. degree in electrical engineering from the University of Illinois at Chicago in 2002. He is currently working on his Ph.D. degree at University of Illinois at Urbana, Champaign under Professor Thomas S. Huang. He has published several papers in the field of machine learning and its ap-

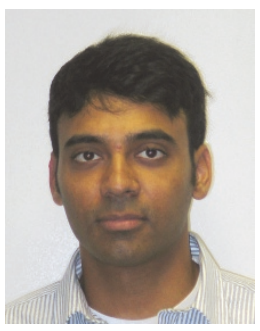
plications in signal processing, computer vision, information retrieval, and other domains.

Thomas S. Huang received his B.S. degree in electrical engineering from National Taiwan University, Taipei, Taiwan, China, and his M.S. and Sc.D. degrees in electrical engineering from the Massachusetts Institute of Technology, Cambridge, Mass. He was on the faculty of the Department of Electrical Engineering at MIT from 1963 to 1973, and on the faculty of the School of Electrical Engineering and Director of its Laboratory for

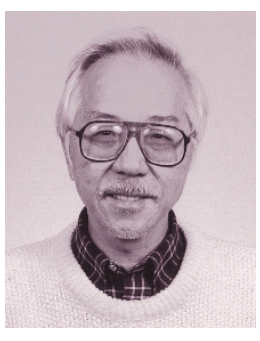
Information and Signal Processing at Purdue University from 1973 to 1980. In 1980, he joined the University of Illinois at UrbanaChampaign, where he is now William L. Everitt Distinguished Professor of Electrical and Computer Engineering, and Research Professor at the Coordinated Science Laboratory, and Head of the Image Formation and Processing Group at the Beckman Institute for Advanced Science and Technology and Cochair of the Institute's major research theme of human-computer intelligent interaction.

Nemanja Petrovic received his Ph.D. degree from University of Illinois in 2004. He is currently a member of research staff at Google Inc., New York. His professional interests are computer vision and machine learning. Dr. Petrovic has published more than 20 papers in the fields of graphical models, video understanding, and data clustering and image enhancement.

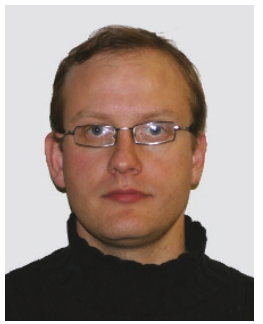

